\title{
Curvas de complacência ou curvas pressão-volume na insuficiência respiratória aguda
}

\author{
SILVIA REGINA RIOS VIEIRA ${ }^{1}$
}

O objetivo do presente trabalho é revisar o uso das curvas de complacência ou curvas pressão-volume (P-V) de pacientes com insuficiência respiratória aguda (IRA) - lesão pulmonar aguda ou acute lung injury (ALI) e síndrome da angústia respiratória aguda (SARA). Foram revisados os principais trabalhos publicados na literatura em língua inglesa, localizados por pesquisa via Medline, que abordavam o uso das curvas P-v na IRA. Idealmente as curvas P-V devem ser realizadas em pacientes com IRA visando uma monitorização e um manejo mais adequado dos mesmos a partir dos dados por elas fornecidos: ponto de inflexão inferior (Pinf), ponto de inflexão superior (Psup) e complacência estática. Todos os métodos disponíveis para a obtenção das mesmas (superseringa, oclusões inspiratórias e fluxo contínuo) são similares desde que fluxos contínuos baixos sejam utilizados e sejam tomados cuidados que permitam o esvaziamento pulmonar antes de realizar as curvas, evitando o surgimento de PEEP (pressão expiratória final positiva) intrínseca. Com estes cuidados o fluxo contínuo torna-se um método simples, não dispendioso, seguro e confiável, para realizar curvas P-V

à beira do leito, facilitando a monitorização dos pacientes com IRA. (J Pneumol 1999;25(6):335-339)

\section{Compliance curves or pressure-volume curves in acute respiratory failure}

The goal of this paper is to review the use of pressure-volume (P-V) Curves or compliance curves in patients with acute respiratory failure (ARF) - acute lung injury (ALI), and acute respiratory distress syndrome (ARDS). The most important papers published in English language literature concerning P-V curves in ARF were found in Medline and reviewed. Ideally, recommendation is made to cal culate P-V curves in all patients with ARF in order to achieve adequate monitoring and management considering

data obtained from the curves, such as lower inflexion point, upper inflexion point, and static compliance. The methods used to obtain P-V curves are supersyringe, multiple inspiratory occlusions and continuous flow. All of them are similar if low continuous flow is used and if care is taken to allow lung emptying and to avoid intrinsic PEEP (positive end expiratory pressure) before accomplishing the

curves. In this way continuous flow can be a simple, inexpensive, secure and reliable method to be carried out at bedside in order to obtain P-V curves that allow for a better monitoring and management of patients with ARF.

Descritores - Lesão pulmonar aguda. Síndrome da angústia respiratória aguda. Curvas pressão-volume. Complacência estática. Ponto de inflexão inferior. Ponto de inflexão superior.

Key words - A cute lung injury. A cute respiratory distress syndrome. Pressure-volume curves. Static compliance. Lower inflexion point. Upper inflexion point.

1. Professora do Centro de Tratamento Intensivo do Hospital de Clínicas de Porto A legre - Departamento de Medicina Interna, UFRGS. Doutora em Medicina pela Universidade Federal do Rio Grande do Sul.

Endereço para correspondência - Silvia Regina Rios Vieira, São Luís, 1.127, A pto. 501 - 90620-170 - Porto Alegre, RS. Tel./ fax (051) 223-4256; E-mail: srvieira@zaz.com.br

Recebido para publicação em 19/2/99. Aprovado, após revisão, em 1/3/99.
Siglas e abreviaturas utilizadas neste trabalho

P-V - Pressão-volume

IRA - Insuficiência respiratória aguda

ALI - Lesão pulmonar aguda

SARA - Síndrome da angústia respiratória aguda

Pinf - Ponto de inflexão inferior

Psup - Ponto de inflexão superior

PEEP - Pressão expiratória final positiva

l/ E - Relação inspiração/ expiração

A curva de complacência ou curva pressão-volume (P-V) é uma técnica utilizada com fins diagnósticos para descrever as propriedades mecânicas estáticas do sistema respiratório, tendo sido adaptada aos pacientes das unidades de tratamento intensivo (UTIS) com o objetivo de avaliar o sistema 
respiratório numa situação extremamente grave ${ }^{(1-4)}$. Sua aplicação costuma ser essencialmente reservada a pacientes com insuficiência respiratória aguda (IRA), incluindo pacientes com síndrome da angústia respiratória aguda (SARA) e lesão pulmonar aguda ou acute lung injury (ALI), situações em que foi proposta como um meio de observar a evolução da gravidade dessa síndrome ${ }^{(5)}$.

A seguir serão feitas considerações sobre fisiologia, forma, aspectos clínicos e técnicas de obtenção das curvas de complacência na IRA.

\section{AsPeCTOS FISIOLÓGICOS}

O deslocamento do sistema respiratório durante a ventilação necessita se opor a forças resistivas, a forças de inércia e a forças elásticas do referido sistema. Essas forças são exercidas sobre a parede torácica e sobre os pulmões. Elas podem ser produzidas pelos músculos respiratórios, como na ventilação espontânea, por ventilador, como na ventilação controlada, ou por ambos, nos casos de ventilação assistida. As patologias alveolares ou parenquimatosas causam essencialmente alterações do componente elástico do sistema respiratório, razão pela qual as curvas pressão-volume são normalmente realizadas em condições estáticas que influenciam na relação pressão-fluxo. 0 sistema respiratório possui igualmente propriedades viscoelásticas, fenômeno esse que corresponde a uma diminuição rápida das forças elásticas de retração para uma mesma pressão. Tais características são observadas facilmente se fizermos uma pausa teleinspiratória e observarmos que, para um mesmo volume pulmonar, a pressão cai em algumas centenas de milissegundos para se equilibrar em um platô (pressão inspiratória final ou pressão de platô). As forças viscoelásticas podem ser consideráveis em pacientes com SARA e devem ser potencialmente consideradas quando se trabalha com as relações pressão-volume. Essa questão das forças viscoelásticas poderá se manifestar principalmente quando as curvas forem analisadas não por um método totalmente estático como o das oclusões inspiratórias, mas por um método de fluxo contínuo lento ${ }^{(6-8)}$.

Um dos problemas de interpretação das curvas pressãovolume do sistema respiratório repousa no fato de que sua análise se baseia na medida da pressão de todo o sistema, dependendo, em parte, das características do pulmão e, em parte, das características da parede. Com efeito, em indivíduos normais, a presença de um ponto de inflexão inferior (Pinf) pode estar presente em decorrência da parede toráci$\mathrm{Ca}^{(9)}$. A lém disso, Mergoni et al. ${ }^{(10)}$ mostraram, recentemente, a influência que podia ser atribuída à parede respiratória nos deslocamentos das curvas modificando suas inclinações, ou seja, o valor absoluto da complacência estática, como também os valores das eventuais inflexões observadas sobre a curva.

\section{FORMA DA CURVA}

Em indivíduos normais, em posição supina, a curva pressão-volume mostra duas inflexões: uma inferior, devido à mecânica da parede torácica quando em baixos volumes pulmonares, e uma superior, devido à hiperdistensão pulmonar em volumes próximos da capacidade pulmonar total(9).

Em pacientes com SARA, a forma habitual da curva pressão-volume é a de uma relação freqüentemente descrita como sigmoidal, com uma parte inferior correspondente a uma complacência baixa, uma parte linear com uma complacência um pouco menos reduzida e uma parte superior onde a complacência cai novamente. A porção inicial da curva é considerada como a que corresponde à abertura de vias aéreas colabadas ${ }^{(11)}$. A parte retilínea da curva é considerada como a que reflete o estado do pulmão após recrutamento alveolar e a avaliação de sua inclinação permite quantificar a gravidade do comprometimento pulmonar. Entre a porção inicial e a porção retilínea está o ponto de inflexão inferior, que corresponde ao nível de pressão a partir do qual ocorre esse recrutamento alveolar ${ }^{(11)}$. A parte superior da curva, que se flete novamente na altura do chamado ponto de inflexão superior (Psup), pode refletir quer o fim do recrutamento alveolar, quer um nível de hiperdistensão(12).

\section{Aspectos CLÍNICOS}

As correlações entre 0 aspecto da curva e o estado da doença foram bem estabelecidas por Matamis et al.(5). Principalmente as relações entre o ponto de inflexão inferior e a eficácia da pressão expiratória positiva têm sido usadas como uma forma de otimização da regulagem do ventilador. Recentemente, estudos de A mato et al. ${ }^{(13,14)}$ estimularam 0 interesse no uso das curvas pressão-volume para o manejo dos pacientes com SARA. Com efeito, esses autores, em estudo prospectivo randomizado, utilizaram a curva pressãovolume para estabelecer os parâmetros ventilatórios e o nível de pressão expiratória final positiva (PEEP) a ser utilizado. Verificaram que o grupo tratado dessa forma e com pequenos volumes correntes, para limitar as pressões de fim de insuflação, apresentou melhora nos parâmetros de oxigenação e na mecânica respiratória, tendo ocorrido redução inicial da mortalidade.

Devido a esses outros possíveis benefícios da compreensão da mecânica respiratória em função das intervenções terapêuticas, torna-se importante, para o manejo de pelo menos alguns pacientes com SARA, a monitorização das curvas pressão-volume. A té 0 momento as técnicas consagradas (superseringa e oclusões inspiratórias), da forma com que são realizadas, geram problemas e/ ou dificultam sua realização rotineira à beira de leito. Por outro lado, as técnicas que utilizam fluxos contínuos, embora mais práticas, ainda não estão devidamente validadas. 
Para atingir-se um ponto ideal dever-se-iam desenvolver técnicas para obtenção das curvas pressão-volume que fossem automatizadas, simplificadas e incorporadas aos apareIhos de ventilação mecânica de forma a facilitar seu uso no manejo dos pacientes com SARA.

Os principais objetivos da realização mais rotineira das curvas pressão-volume na SARA seriam calcular e monitorizar a complacência estática do sistema respiratório ao longo da evolução da doença; verificar a presença de um ponto de inflexão inferior e utilizá-lo, quando presente, para estabelecer o valor ideal de PEEP a ser empregado; verificar a presença de um ponto de inflexão superior e adotá-lo, quando presente, como controle das pressões de via aérea a serem utilizadas, numa tentativa de evitar hiperdistensão alveolar.

\section{TÉCNICAS DE OBTENÇÃO DAS CURVAS}

Várias técnicas têm sido empregadas para obter as curvas pressão-volume:

Técnica de superseringa - É a técnica mais utilizada, mais conhecida e mais antiga, sendo considerada como referência para traçar as curvas pressão-volume estáticas do sistema respiratório em pacientes com IRA ${ }^{(5)}$. Consiste em insuflar por patamares volumétricos sucessivos até $1,5 \mathrm{~L}$ ou $2 \mathrm{~L}$ em inspiração e em desinflar nos mesmos patamares em expiração. Essa técnica foi largamente popularizada pelos trabaIhos de Matamis e Lemaire e permitiu descrever o aspecto da curva em diferentes estágios da doença( ${ }^{(5)}$. Numerosas limitações e artefatos são associados a esse método ${ }^{(15)}$. U m de seus grandes inconvenientes é a desconexão obrigatória do paciente do ventilador. Um outro problema é o tempo necessário para a realização de toda a curva, pois a manobra de inflação requer 45 a 60 segundos. A lém disso, está associada a perda de volume, já que o consumo de oxigênio $\left(\mathrm{O}_{2}\right)$ no interior do pulmão é mais rápido do que a produção de dióxido de carbono $\left(\mathrm{CO}_{2}\right)$. Isso faz com que a medida do volume pela seringa seja superestimada e cause alterações principalmente no ramo expiratório da curva. Esse fato foi bem demonstrado por Dall'Ava-Santucci et al. ${ }^{(16,17)}$, quando compararam o volume medido pelo deslocamento da seringa com mudanças de volume torácico obtidas a partir de pletismografia.

Técnica das oclusões inspiratórias múltiplas - Essa técnica, bastante empregada nas determinações das curvas pressão-volume estáticas, foi introduzida por diferentes autores ${ }^{(18,19)}$. Um dos primeiros estudos foi o de Levy et al. ${ }^{(18)}$ que, utilizando um ventilador munido de oclusão teleinspiratória e teleexpiratória e empregando volumes progressivamente crescentes, mostrou que era possível, sob certas condições técnicas, traçar o conjunto da curva pressão-volume. Desde então, o método tem sido largamente utilizado, com as vantagens de não necessitar desconectar 0 doente do ventilador e de poder ser realizado a partir de qualquer nível de base, sendo efetuado mais facilmente em PEEP(19-21). A principal desvantagem é o longo tempo gasto em sua realização, de aproximadamente 15 minutos, motivo pelo qual é usada em pesquisa, mas torna-se trabalhosa para ser empregada no dia-a-dia à beira do leito.

Técnica de insuflação com fluxo contínuo - U m método alternativo, usando um fluxo contínuo, foi também proposto para determinar a complacência respiratória(22). Esse método dinâmico, embora introduzido há vários anos, ainda não foi devidamente validado. Baseia-se no princípio de que, quando um fluxo constante penetra nos pulmões, a razão de mudança das pressões é inversamente proporcional à complacência do sistema respiratório. Durante o procedimento, dois segmentos podem ser identificados na curva de pressão da via aérea: a primeira porção da curva de pressão está relacionada às propriedades resistivas do sistema respiratório; a segunda porção, caracterizada por um aumento linear na pressão da via aérea a uma razão inversamente proporcional à complacência, está relacionada às propriedades elásticas do sistema respiratório. Suratt e 0 wens ${ }^{(23)}$ compararam o método de fluxo contínuo com o estático e demonstraram que os valores de complacência medidos com ambos os métodos eram fortemente correlacionados. A técnica foi novamente testada por Mankikian ${ }^{(7)}$, usando um fluxo contínuo lento de 1,7L. $\mathrm{min}^{-1}$, sendo que as curvas eram superponíveis às obtidas com a superseringa. Sua principal vantagem era a de não modificar o volume pulmonar antes de efetuar a manobra. Ranieri et al. ${ }^{(20)}$ retomaram a técnica, tendo observado que representava uma ferramenta simples e não invasiva capaz de detectar e predizer os efeitos da PEEP no recrutamento alveolar. N esse estudo, em que foi adotado um fluxo constante alto, variando de 20 a $60 \mathrm{~L}$. $\mathrm{min}^{-1}$, os pontos de inflexão inferior e superior não podiam ser corretamente determinados. Recentemente a técnica foi novamente empregada no trabalho de Servillo et al. ${ }^{(8)}$. Utilizando um fluxo inspiratório constante de $15 \mathrm{~L}$. $\mathrm{min}^{-1}$, os autores verificaram que 0 método era de fácil aplicabilidade e com vantagens práticas. No entanto as curvas assim obtidas eram desviadas para a direita devido às propriedades resistivas e elásticas do sistema respiratório, resultando assim em uma hiperestimação dos pontos de inflexão inferior.

Comparação entre os métodos - Recentemente realizamos um trabalho ${ }^{(24)}$ com o objetivo de comparar, à beira do leito, em pacientes com IRA, os três métodos para medida das complacências toracopulmonar, pulmonar e torácica: os tradicionais métodos da superseringa e das oclusões inspiratórias com uma técnica mais nova, simples, rápida e pouco dispendiosa, que não desconecta o paciente do ventilador e usa fluxos constantes mais baixos (por exemplo 3 e $9 \mathrm{~L}$. $\mathrm{min}^{-1}$ ) liberados por um ventilador convencional.

Neste trabalho ${ }^{(24)}$ foram estudados, prospectivamente, 14 pacientes com diagnóstico de ALI OU SARA, submetidos à realização de curvas pressão-volume toracopulmonares, pulmo- 
nares e torácicas, obtidas pelo método da superseringa ${ }^{(2,5,15)}$, das oclusões inspiratórias múltiplas ${ }^{(18,19)}$ e de dois fluxos contínuos: 3 e $6 \mathrm{~L} \cdot \mathrm{min}^{-1}$. Estes fluxos foram liberados pelo ventilador César usando-se os seguintes parâmetros: freqüência respiratória de 5, relação inspiração/ expiração (I/E) de $80 \%$ (tempo inspiratório de 9,6 segundos) e volumes correntes de $500 \mathrm{ml}$ para o fluxo $3 \mathrm{~L}$. $\mathrm{min}^{-1}$ e 1.500 para o fluxo $9 \mathrm{~L}$. $\mathrm{min}^{-1}$.

Os resultados deste estudo ${ }^{(24)}$ demonstraram que as curvas pressão-volume toracopulmonares, pulmonares e torácicas obtidas com os métodos das superseringa, das oclusões inspiratórias múltiplas e do fluxo contínuo $3 \mathrm{~L}$. $\mathrm{min}^{-1}$ foram superponíveis. 0 método do fluxo contínuo com $9 \mathrm{~L}$. $\mathrm{min}^{-1}$ foi associado com um leve desvio da curva para a direita, provavelmente devido a um componente resistivo. Os valores do ponto de inflexão inferior e das complacências toracopulmonares, pulmonares e torácicas não foram significativamente diferentes entre os métodos. Este estudo indicou que a técnica do fluxo contínuo pode avaliar corretamente as propriedades elásticas do pulmão e da parede torácica, sem ser afetado pelas propriedades resistivas do tubo endotraqueal e do sistema respiratório, principalmente quando um fluxo contínuo baixo, como 3L. $\mathrm{min}^{-1}$, foi usado.

A resistência do sistema respiratório é parcialmente relacionada ao nível de fluxo inspiratório ${ }^{25,26)}$. Servillo et al. ${ }^{(8)}$ verificaram que as curvas pressão-volume desviavam-se para a direita quando um fluxo constante de $15 \mathrm{~L}$. $\mathrm{min}^{-1}$ era liberado pelo ventilador. Em contraste, Mankikian et al..$^{(7)}$ observaram que as curvas obtidas com o fluxo constante de 1,7L. $\mathrm{min}^{-1}$ eram superponíveis às traçadas com o método da superseringa. N ossos resultados (24, em uma série de pacientes com resistência respiratória normal ou levemente aumentada, demonstraram que as curvas pressão-volume obtidas com um fluxo contínuo de $3 \mathrm{~L}$. $\mathrm{min}^{-1}$ foram similares àquelas dos métodos estáticos, enquanto as obtidas com um fluxo contínuo de $9 \mathrm{~L}$. min $^{-1}$ mostraram um leve desvio para a direita. Tal desvio não influenciou significativamente a medida das complacências e dos pontos de inflexão inferior, já que foi observada uma boa concordância para os valores destas medidas tanto nas curvas toracopulmonares, como nas pulmonares e nas torácicas obtidas por todos os métodos. A lém disso, nossos resultados confirmaram a idéia de que a resistência do sistema respiratório é fluxo-dependente(27). Em nosso estudo, pacientes com doença pulmonar obstrutiva crônica (DPOC) e broncoespasmo foram excluídos, sendo necessárias novas investigações para definir o nível ótimo de fluxo a ser utilizado em pacientes com elevada resistência respiratória.

0 método da superseringa é tradicionalmente considerado como o de referência para medir as curvas pressão-volu$m \mathrm{e}^{(5)}$, tendo como inconvenientes a desconexão obrigatória do paciente do ventilador e problemas associados à perda de volume devido ao consumo de oxigênio pelos pulmões ${ }^{(15,16)}$. A técnica das oclusões inspiratórias também é bastante con- fiável, pode ser realizada sem a desconexão do ventilador, é amplamente utilizada em trabalhos de pesquisa, principalmente por permitir sua fácil realização em PEEP(18-21), mas tem como desvantagem o longo tempo necessário para sua realização. 0 fluxo contínuo, por sua vez, apresentou várias vantagens em relação aos outros métodos: 1) não houve necessidade de desconectar o paciente do ventilador; 2 ) as manobras totais de reconstrução das curvas pressão-volume foram rápidas e permitiram a construção simultânea das curvas pressão-volume na tela do respirador; 3 ) o método foi simples, não dispendioso e não necessitou de equipamentos específicos; 4) a análise das curvas pressão-volume pôde ser realizada à beira de leito, já que 0 ventilador era equipado com um programa de computador que permitia a visibilização da curva em sua tela e possibilitava as medidas da inclinação da curva e do ponto de inflexão inferior, com o auxílio de cursores móveis. A lém disso, desde que o ventilador fosse equipado com um transdutor de pressão adicional que pudesse ser conectado à extremidade distal da traquéia, a curva pressão-volume podia ser registrada à beira do leito sem a influência das propriedades resistivas dos circuitos ventilatórios e do tubo endotraqueal. Embora essa técnica para a medida da curva pressão-volume tenha sido testada com 0 ventilador César, ela pode ser implementada com vários ventiladores de UTI, desde que equipados com uma tela e com um programa que permitam visibilizar e analisar as curvas pressão-volume.

0 método apresentou, porém, algumas limitações. Primeiramente, o fluxo inspiratório foi indiretamente estabelecido dependendo da razão $\mathrm{I} / \mathrm{E}$, da freqüência respiratória e do volume corrente. Em segundo lugar, a obtenção de um fluxo inspiratório constante por um período superior a dez segundos não foi possível devido a uma freqüência respiratória mínima que teve de ser utilizada e que, nos ventiladores atualmente em uso, é igual ou superior a cinco ciclos por minuto. Como conseqüência, os volumes correntes máximos que puderam ser utilizados ficaram limitados a $500 \mathrm{ml}$ para um fluxo de $3 \mathrm{~L}$. $\mathrm{min}^{-1}$ ou a $1.500 \mathrm{ml}$ para um fluxo de $9 \mathrm{~L}$. $\mathrm{min}^{-1}$. Assim sendo, o ponto de inflexão superior não foi determinado com essa técnica em pacientes com IRA moderada nos quais volumes mais altos deveriam ser alcançados. Essas dificuldades poderiam, talvez, ser minimizadas se os ventiladores viessem equipados com um gerador de fluxo que fornecesse fluxos contínuos de 3, 6 e $9 \mathrm{~L} \cdot \mathrm{min}^{-1}$ durante períodos de administração de 10, 20 e 30 segundos. Seria também interessante que eles tivessem um botão que permitisse realizar uma expiração prolongada antes da manobra, além de uma tela e de um programa de computador para visibilização e análise da curva pressão-volume. Dessa forma poderia haver uma melhora importante da monitorização respiratória à beira do leito. Tornar a curva pressão-volume facilmente acessível aos intensivistas, sem desconectar o paciente do ventilador, representaria, certamente, um avanço no campo da 
ventilação mecânica. Enquanto tais aprimoramentos técnicos são aguardados, o método descrito neste estudo (24) constitui uma alternativa que pode ser adotada na realização das curvas pressão-volume.

\section{CONCLUSÕES}

As curvas pressão-volume devem ser realizadas, sempre que possível, visando uma melhor monitorização e manejo de pacientes com IRA, a partir dos dados que fornecem (Pinf, Psup e complacência estática). Estas curvas podem ser realizadas por diferentes métodos. Todos os métodos disponíveis para obtenção das curvas pressão-volume (superseringa, oclusões inspiratórias múltiplas e fluxo contínuo) são similares desde que fluxos contínuos baixos sejam utilizados (como abaixo de $9 \mathrm{~L} \cdot \mathrm{min}^{-1}$ ) e desde que cuidados sejam tomados para permitir esvaziamento pulmonar antes da obtenção das curvas e evitar PEEP intrínseca. Com estes cuidados o fluxo contínuo torna-se um método simples, seguro, confiável e não dispendioso para realizar as curvas à beira do leito. Porém, para trabalhos de pesquisa, principalmente aqueles que necessitam realizar curvas pressão-volume em diferentes níveis de PEEP, continua-se empregando o conhecido método das oclusões inspiratórias que, embora mais difícil, mais demorado e menos prático, possibilita uma correção mais adequada dos níveis de PEEP extrínseca e intrínseca.

\section{REFERÊNCIAS}

1. Falke KJ, Pontoppidan H, Kumar A, Leith DE, Geffin B, Laver MB. Ventilation with end-expiratory pressure in acute lung disease. J Clin Invest 1972;51:2315-2323.

2. Harf A, Lemaire F, Lorino $H$, Atlan G. Etude de la mecanique ventilatoire: application à la ventilation artificielle. Bull Eur Physiopathol Respir 1975;11:709-729.

3. Suter PM, Fairley BF, Isenberg MD. Optimum end-expiratory airway pressure in patients with acute pulmonary failure. N Engl J Med 1975; 292:284-289.

4. Bone RC. Diagnosis of causes for acute respiratory distress by pressure-volume curves. Chest 1976;70:740-746.

5. Matamis D, Lemaire F, H arf A, Brun-Buisson C, Ansquer J C, A tlan G. Total respiratory pressure-volume curves in the adult respiratory distress syndrome. Chest 1984;86:58-66.

6. Holzapfel L, Robert D, Perrin F, Blanc PL, Palmier B, Guerin C. Static pressure-volume curves and effect of positive end expiratory pressure on gas exchange in adult respiratory distress syndrome. C rit Care Med 1983;11:591-598.

7. Mankikian B, Lemaire $F$, Benito $S, B$ run-Buisson $C, H$ arf $A$, Maillot $J$ J, Becker J. A new device for measurement of pulmonary pressurevolume curves in patients on mechanical ventilation. Crit Care Med 1983;11:897-901.

8. Servillo G, Svantesson C, Beydon L, Roupie E, Brochard L, Lemaire $\mathrm{F}$, J onson B. Pressure-volume curves in Acute Respiratory Failure. Automated low flow inflation versus occlusion. Am J Resp Crit Care Med 1997;155:1629-1636.

9. Agostoni E, Hyatt RE. Static behavior of the respiratory system. In: Macklem PT, Mead J, eds. Handbook of physiology. Bethesda, Maryland: American Physiological Society, 1986;113-130.

10. Mergoni M, Martelli A, Volpi A, Primavera S, Zuccoli P, Rossi A. Impact of positive end-expiratory pressure on chest wall and lung pres- sure-volume curve in acute respiratory failure. Am J Resp Crit Care Med 1997;156:846-854.

11. Gattinoni L, Pesenti A, Avalli L, Rossi F, Bombino M. Pressure-volume curve of total respiratory system in acute respiratory failure. Computed tomographic scan study. Am Rev Resp Dis 1987;136:730-736.

12. Roupie E, Dambrosio M, Servillo G, Mentec H, El Atrous S, Beydon $L$, Brun-Buisson C, Lemaire F, Brochard L. Titration of tidal volume and induced hypercapnia in acute respiratory distress syndrome. Am J Resp Crit Care Med 1995;152:121-128.

13. A mato MBP, Barbas CSV, Medeiros DM, Schettino GDP, Lorenzi FiIho G, Kairalla RA, Deheinzelin D, Morais C, Fernandes EO, Takagak $Y T$, Carvalho CRR. Beneficial effects of the "open lung approach" with low distending pressures in acute respiratory distress syndrome. Am J Resp Crit Care Med 1995;152:1835-1846.

14. A mato MBP, Barbas CSV, Medeiros DM, Magaldi RB, Schettino GPB, Lorenzi-Filho G, Kairalla RA, Deheinzelin D, Munoz C, Oliveira R, Takagaki TY, Carvalho CRR. Effect of a protective-ventilation strategy on mortality in the acute respiratory distress syndrome. $\mathrm{N}$ Engl J Med 1998;338:347-354

15. Gattinoni L, Mascheroni D, Basilico E, Foti G, Pesenti A, Avalli $L$. Volume/ pressure curve of total respiratory system in paralyzed patients: artifacts and correction factors. Intensive Care Med 1987;13: 19-25.

16. Dall'Ava-Santucci J , Armaganidis A, Brunet F. Causes of error of respiratory pressure-volume curves in paralyzed subjects. J Appl Physiol 1988;64:42-49.

17. Dall'ava-Santucci J A Armaganidis A, B runet F, Dhainaut J F, Nouira S, Morisseau D, Lockhart A. Mechanical effects of PEEP in patients with adult respiratory distress syndrome. J A ppl Physiol 1990;68:843-848.

18. Levy P, Similowski T, Corbeil C, Albala M, Pariente R, Milic-Emili J, J onson B. A method for studying the static volume-pressure curves of the respiratory system during mechanical ventilation. J Crit Care 1989; 4:83-89.

19. Ranieri VM, Eissa NT, Corbeil C, Chassé M, Braidy J, Matar N, MilicEmili J. Effects of positive end-expiratory pressure on alveolar recruitment and gas exchange in patients with the adult respiratory distress syndrome. Am Rev Resp Dis 1991;144:544-551.

20. Ranieri VM, Giuliani R, Flore T, Dambrosio M, Milic-Emili J. Volumepressure curve of the respiratory system predicts effects of PEEP in ARDS: "Occlusion" versus "constant flow" technique. Am J Respir Crit Care Med 1994;149:19-27.

21. Ranieri VM, Mascia LM, Fiore T, Bruno F, Brienza A, Giuliani R. Cardiorespiratory effects of positive end-expiratory pressure during progressive tidal volume reduction (permissive hypercapnia) in patients with acute respiratory distress syndrome. A nesthesiology 1995;83:710 720 .

22. Suratt $P M, O$ wens $D H$, Kilgore WT, Harry RR, Hsiao HS. A pulse method of measuring respiratory system compliance. J Appl Physiol 1980;49:1116-1121.

23. Suratt $P M, O$ wens $D H$. A pulse method of measuring respiratory system compliance in ventilated patients. Chest 1981;80:34-38.

24. Lu Q, Vieira SRR, Richecoeur J, Puybasset L, Kalfon P, Coriat P, Rouby JJ. A simple automated method for measuring pressure-volume curves during mechanical ventilation. Am J Respir Crit Care Med 1999;159:275-282.

25. Polese G, Rossi A, Appendini L, Brandi G, Bates J HT, Brandolese R. Partitioning of respiratory mechanics in mechanically ventilated patients. J Appl Physiol 1991;71:2425-2433.

26. Tantucci C, Corbeil C, Chasse M, Robatto FM, Nava S, Braidy J, Matar N, Milic-Emili J. Flow and volume dependence of respiratory system. Flow resistance in patients with adult respiratory distress syndrome. Am Rev Resp Dis 1992;145:355-360.

27. D'Angelo $E$, Calderini $E$, Torri $E$, Robatto $M$, Bono D, Milic-Emili J Respiratory mechanics in anesthetized paralyzed humans: effects of flow, volume, and time. J Appl Physiol 1989;67:2556-2564. 